\title{
THE SCHOUTEN-VAN KAMPEN AFFINE CONNECTION ADAPTED TO AN ALMOST (PARA) CONTACT METRIC STRUCTURE
}

\author{
Zbigniew Olszak
}

\begin{abstract}
We study the Schouten-van Kampen connection associated to an almost contact or paracontact metric structure. With the help of such a connection, some classes of almost (para) contact metric manifolds are characterized. Certain curvature properties of this connection are found.
\end{abstract}

\section{Introduction}

The Schouten-van Kampen connection is one of the most natural connections adapted to a pair of complementary distributions on a differentiable manifold endowed with an affine connection; cf. $[\mathbf{4}, \mathbf{1 1}, \mathbf{2 2}]$, etc. We would like to pay much attention to the papers $[\mathbf{2 3}]-[\mathbf{2 6}]$ by A. F. Solov'ev, who has investigated hyperdistributions in Riemannian manifolds using the Schouten-van Kampen connection.

On the other hand, any almost contact as well as paracontact metric manifold admits a hyperdistribution. Such distributions and some kinds of affine connections adapted to these distributions were studied by many authors; see $[\mathbf{2}, \mathbf{3}, \mathbf{5}, \mathbf{6}, \mathbf{1 4}$, 15, 21, 27, 28], etc.

In this short note, we are interested in Schouten-van Kampen connections which are associated to the hyperdistributions occuring on almost contact as well as paracontact (possibly indefinite) metric manifolds. With the help of the Schouten-van Kampen connection, we characterize some classes of almost (para) contact metric manifolds, and find certain curvature properties of this connection on these manifolds.

\section{Hyperdistributions in pseudo-Riemannian manifolds}

Let $M$ be a (connected) pseudo-Riemannian manifold of an arbitrary signature $(p, n-p), 0 \leqslant p \leqslant n, n=\operatorname{dim} M \geqslant 2$. By $g$ will be denoted the pseudo-Riemannian metric on $M$, and by $\nabla$ the Levi-Civita connection coming from the metric $g$.

2010 Mathematics Subject Classification. Primary 53C25; Secondary 53C07, 53C50, 53D15.

Key words and phrases. Distribution, Schouten-van Kampen affine connection, almost (para) contact metric manifold, (para) contact distribution. 
Assume that $\mathcal{H}$ and $\mathcal{V}$ are two complementary, orthogonal distributions on $M$ such that $\operatorname{dim} \mathcal{H}=n-1, \operatorname{dim} \mathcal{V}=1$, and the distribution $\mathcal{V}$ is non-null. Thus, $T M=\mathcal{H} \oplus \mathcal{V}, \mathcal{H} \cap \mathcal{V}=\{0\}$ and $\mathcal{H} \perp \mathcal{V}$. Asume that $\xi$ is a unit vector field and $\eta$ is a linear form such that $\eta(\xi)=1, g(\xi, \xi)=\varepsilon= \pm 1$ and

$$
\mathcal{H}=\operatorname{ker} \eta, \quad \mathcal{V}=\operatorname{span}\{\xi\} .
$$

We can always choose such $\xi$ and $\eta$ at least locally (in a certain neighborhood of an arbitrary chosen point of $M)$. Then, we also have $\eta(X)=\varepsilon g(X, \xi)$. Moreover, it holds that $\nabla_{X} \xi \in \mathcal{H}$.

For any $X \in T M$, by $X^{h}$ and $X^{v}$ we denote the projections of $X$ onto $\mathcal{H}$ and $\mathcal{V}$, respectively. Thus, we have $X=X^{h}+X^{v}$ with

$$
X^{h}=X-\eta(X) \xi, \quad X^{v}=\eta(X) \xi .
$$

The Schouten-van Kampen connection $\widetilde{\nabla}$ associated to the Levi-Civita connection $\nabla$ and adapted to the pair of distributions $(\mathcal{H}, \mathcal{V})$ is defined by (cf. e.g. $[4])$

$$
\widetilde{\nabla}_{X} Y=\left(\nabla_{X} Y^{h}\right)^{h}+\left(\nabla_{X} Y^{v}\right)^{v}
$$

and the corresponding second fundamental form $B$ is defined by $B=\nabla-\widetilde{\nabla}$. Note that the condition (2.3) implies the parallelity of the distributions $\mathcal{H}$ and $\mathcal{V}$ with respect to the Schouten-van Kampen connection $\widetilde{\nabla}$.

Having (2.2), one can compute

$$
\begin{aligned}
& \left(\nabla_{X} Y^{h}\right)^{h}=\nabla_{X} Y-\eta\left(\nabla_{X} Y\right) \xi-\eta(Y) \nabla_{X} \xi \\
& \left(\nabla_{X} Y^{v}\right)^{v}=\left(\left(\nabla_{X} \eta\right)(Y)+\eta\left(\nabla_{X} Y\right)\right) \xi
\end{aligned}
$$

which enables us to express the Schouten-van Kampen connection with the help of the Levi-Civita connection in the following way (cf. [23])

$$
\widetilde{\nabla}_{X} Y=\nabla_{X} Y-\eta(Y) \nabla_{X} \xi+\left(\nabla_{X} \eta\right)(Y) \xi
$$

Thus, the second fundamental form $B$ and the torsion $\widetilde{T}$ of $\widetilde{\nabla}$ are (cf. $[\mathbf{2 3}]-[\mathbf{2 4}]$ )

$$
\begin{aligned}
& B(X, Y)=\eta(Y) \nabla_{X} \xi-\left(\nabla_{X} \eta\right)(Y) \xi \\
& \widetilde{T}(X, Y)=\eta(X) \nabla_{Y} \xi-\eta(Y) \nabla_{X} \xi+2 d \eta(X, Y) \xi .
\end{aligned}
$$

The linear operator $L$ defined by

$$
L X=-\nabla_{X} \xi
$$

will be called the shape operator. It can be noticed that $\xi$ is a Killing vector field if anf only if $L$ is an antisymmetric operator. From (2.5), we see that $B$ can be described with the help of the shape operator $L$, namely

$$
B(X, Y)=-\eta(Y) L X+\varepsilon g(L X, Y) \xi .
$$

Moreover, from (2.5) and (2.6), we deduce that $\widetilde{T}=-2 \mathcal{A}(B), \mathcal{A}$ being the antisymmetrization operation. 
With the help of the Schouten-van Kampen connection (2.4), many properties of some geometric objects connected with the distributions $\mathcal{H}, \mathcal{V}$ can be characterized (cf. [23] - [25]). Probably, the most spectacular is the following statement: $g$, $\xi$ and $\eta$ are parallel with respect to $\widetilde{\nabla}$, that is, $\widetilde{\nabla} \xi=0, \widetilde{\nabla} g=0, \widetilde{\nabla} \eta=0$.

We finish this section with the following statement:

THEOREM 2.1. The Schouten-van Kampen connection $\widetilde{\nabla}$ associated to the LeviCivita connection $\nabla$ and adapted to the pair (2.1) is just the only one affine connection, which is metric and its torsion is of the form (2.6).

ProOF. It remains to prove that if an affine connection $\widetilde{\nabla}$ is metric $(\widetilde{\nabla} g=0)$ and its torsion is given by (2.6), then it is given by (2.4). For, recall the famous result stating that any metric connection can be expressed with the help of its torsion $\widetilde{T}$ in the following way

$$
\begin{aligned}
g\left(\widetilde{\nabla}_{X} Y, Z\right)= & g\left(\nabla_{X} Y, Z\right) \\
& +\frac{1}{2}(g(\widetilde{T}(X, Y), Z)-g(\widetilde{T}(X, Z), Y)-g(\widetilde{T}(Y, Z), X)) .
\end{aligned}
$$

Applying (2.6) into the above relation enables us to deduce the following

$$
g\left(\widetilde{\nabla}_{X} Y, Z\right)=g\left(\nabla_{X} Y, Z\right)-\eta(Y) g\left(\nabla_{X} \xi, Z\right)+\eta(Z) g\left(\nabla_{X} \xi, Y\right),
$$

which immediatelly leads to (2.4).

\section{The curvature of the Schouten-van Kampen connection}

We keep the assumptions and notations from the previous section.

Let $\widetilde{R}$ and $R$ be the curvature operators of the Levi-Civita connection $\nabla$ and the Schouten-van Kampen connection $\widetilde{\nabla}$,

$$
\widetilde{R}(X, Y)=\left[\widetilde{\nabla}_{X}, \widetilde{\nabla}_{Y}\right]-\widetilde{\nabla}_{[X, Y]}, \quad R(X, Y)=\left[\nabla_{X}, \nabla_{Y}\right]-\nabla_{[X, Y]} .
$$

Using (2.4), by direct calculations, we obtain the following formula connecting $\widetilde{R}$ and $R$ (cf. [23])

$$
\begin{aligned}
\widetilde{R}(X, Y) Z= & R(X, Y) Z-\eta(R(X, Y) Z) \xi-\eta(Z) R(X, Y) \xi \\
& +\left(\nabla_{Y} \eta\right)(Z) \nabla_{X} \xi-\left(\nabla_{X} \eta\right)(Z) \nabla_{Y} \xi .
\end{aligned}
$$

We can write the formula (3.1) with the help of the shape operator $L$. To do it, we need to use the following consequences of (2.7)

$$
\begin{aligned}
\left(\nabla_{X} \eta\right)(Y) & =-\varepsilon g(L X, Y), \\
R(X, Y) \xi & =-\left(\nabla_{X} L\right) Y+\left(\nabla_{Y} L\right) X, \\
\eta(R(X, Y) Z) & =\varepsilon g\left(\left(\nabla_{X} L\right) Y-\left(\nabla_{Y} L\right) X, Z\right) .
\end{aligned}
$$

Now, applying the above relations and (2.7) into (3.1), we obtain the following 
TheOREM 3.1. The curvature operators $\widetilde{R}$ and $R$ are related by

$$
\begin{aligned}
\widetilde{R}(X, Y) Z= & R(X, Y) Z-\varepsilon g\left(\left(\nabla_{X} L\right) Y-\left(\nabla_{Y} L\right) X, Z\right) \xi \\
& +\eta(Z)\left(\left(\nabla_{X} L\right) Y-\left(\nabla_{Y} L\right) X\right) \\
& +\varepsilon g(L Y, Z) L X-\varepsilon g(L X, Z) L Y .
\end{aligned}
$$

We will also consider the Riemann curvature $(0,4)$-tensors $\widetilde{R}, R$, and the Ricci curvature tensors $\widetilde{S}, S$, and the scalar curvatures $\widetilde{r}, r$ of the connections $\widetilde{\nabla}$ and $\nabla$ defined by

$$
\begin{array}{cl}
\widetilde{R}(X, Y, Z, W)=g(\widetilde{R}(X, Y) Z, W), & R(X, Y, Z, W)=g(R(X, Y) Z, W), \\
\widetilde{S}(Y, Z)=\operatorname{tr}\{X \mapsto \widetilde{R}(X, Y) Z\}, & S(Y, Z)=\operatorname{tr}\{X \mapsto R(X, Y) Z\}, \\
\widetilde{r}=\operatorname{tr}_{g}\{(Y, Z) \mapsto \widetilde{S}(Y, Z)\}, & r=\operatorname{tr}_{g}\{(Y, Z) \mapsto S(Y, Z)\} .
\end{array}
$$

Having (3.3), we obtain by

COROLlary 3.1. The Riemann curvature $(0,4)$-tensors $\widetilde{R}$ and $R$ are related

$$
\begin{aligned}
\widetilde{R}(X, Y, Z, W)= & R(X, Y, Z, W)-g\left(\left(\nabla_{X} L\right) Y-\left(\nabla_{Y} L\right) X, Z\right) \eta(W) \\
& +g\left(\left(\nabla_{X} L\right) Y-\left(\nabla_{Y} L\right) X, W\right) \eta(Z) \\
& +\varepsilon g(L X, W) g(L Y, Z)-\varepsilon g(L X, Z) g(L Y, W) .
\end{aligned}
$$

Note that from (3.4), the skew-symmetry of $\widetilde{R}$ with respect to the last two arguments follows additionally, that is, $\widetilde{R}(X, Y, Z, W)=-\widetilde{R}(X, Y, W, Z)$.

It is also worthwhile to notice that $\eta(L Y)=\varepsilon g(L Y, \xi)=0$ by $(2.7)$. Hence, for the covariant derivative $\nabla L$, we deduce

$$
g\left(\left(\nabla_{X} L\right) Y, \xi\right)=g(L X, L Y) .
$$

Some additional consequences of (3.1) can be stated as it follows.

Corollary 3.2. The Ricci curvature tensors $\widetilde{S}$ and $S$ are related by

$$
\begin{aligned}
\widetilde{S}(Y, Z)= & S(Y, Z)-\varepsilon R(\xi, Y, Z, \xi)-S(\xi, Y) \eta(Z) \\
& +\left(\nabla_{Y} \eta\right)(Z) \operatorname{div} \xi-\left(\nabla_{\nabla_{Y} \xi} \eta\right)(Z),
\end{aligned}
$$

where $\operatorname{div} \xi=\operatorname{tr}\left\{X \mapsto \nabla_{X} \xi\right\}$ is the divergence of the vector field $\xi$.

COROLlARY 3.3. The scalar curvatures $\widetilde{r}$ and $r$ are related by

$$
\widetilde{r}=r-2 \varepsilon S(\xi, \xi)+\varepsilon(\operatorname{div} \xi)^{2}-\operatorname{tr}_{g}\left\{(Y, Z) \mapsto\left(\nabla_{\nabla_{Y} \xi} \eta\right)(Z)\right) .
$$

As usually, a non-degenerate section $\sigma$ is an arbitrary 2-dimensional subspace of a tangent space $T_{p} M, p \in M$, such that $\left.g\right|_{\sigma}$ is of algebraic rank 2 . The sectional curvatures for $\nabla$ and $\widetilde{\nabla}$ are defined in the standard way

$$
\begin{aligned}
& K(\sigma)=R(X, Y, Y, X) \cdot\left(g(X, X) g(Y, Y)-g^{2}(X, Y)\right)^{-1}, \\
& \widetilde{K}(\sigma)=\widetilde{R}(X, Y, Y, X) \cdot\left(g(X, X) g(Y, Y)-g^{2}(X, Y)\right)^{-1},
\end{aligned}
$$

where the pair $X, Y$ is a basis in $\sigma$. 
COROLlary 3.4. The sectional curvatures $\widetilde{K}$ and $K$ are related by

$$
\begin{aligned}
\widetilde{K}(\sigma)= & K(\sigma)+\left(g(X, X) g(Y, Y)-g^{2}(X, Y)\right)^{-1} . \\
& \cdot(-\eta(X) R(X, Y, Y, \xi)+\eta(Y) R(Y, X, X, \xi) \\
& \left.+\varepsilon\left(\nabla_{X} \eta\right)(X)\left(\nabla_{Y} \eta\right)(Y)-\varepsilon\left(\nabla_{X} \eta\right)(Y)\left(\nabla_{Y} \eta\right)(X)\right),
\end{aligned}
$$

where the pair $X, Y$ is a basis of a non-degenerate section $\sigma$.

\section{Almost (para) contact metric manifolds}

In the geometric literature, we find various classes of almost contact or paracontact metric structures; see e.g. $[\mathbf{6}, \mathbf{7}, \mathbf{8}, \mathbf{1 0}, \mathbf{1 3}, \mathbf{2 9}, \mathbf{3 2}]$, etc. The following convention unifying both the contact and paracontact notations seems to be useful for our purposes. It is a small generalization of the idea applied by S. Erdem in $[\mathbf{1 0}]$.

Let $M$ be a $(2 n+1)$-dimensional (connected) differentiable manifold endowed with a quadruplet $(\varphi, \xi, \eta, g)$, where $\varphi$ is $(1,1)$-tensor field, $\xi$ is a vector field, $\eta$ is a 1-form, and $g$ is a pseudo-Riemannian such that

$$
\begin{gathered}
\varphi^{2} X=\mu(X-\eta(X) \xi), \quad \eta(\xi)=1, \\
g(\varphi X, \varphi Y)=-\mu(g(X, Y)-\varepsilon \eta(X) \eta(Y)),
\end{gathered}
$$

where $\varepsilon, \mu= \pm 1$. As a consequence of the above conditions, we have additionally

$$
\varphi \xi=0, \quad \eta \circ \varphi=0, \quad \eta(X)=\varepsilon g(X, \xi), \quad g(\xi, \xi)=\varepsilon .
$$

The manifold $M$ will be called almost (para) contact metric, and the quadruplet $(\varphi, \xi, \eta, g)$ will be called the almost (para) contact metric structure on $M$. For such a manifold, the fundamental 2 -form $\Phi$ (a skew-symmetric $(0,2)$-tensor field of maximal algebraic rank $(=2 n))$ is defined by $\Phi(X, Y)=g(X, \varphi Y)$.

When $\mu=-1$, then the manifold $M$ is an almost contact metric manifold. In this case the metric $g$ is assumed to be pseudo-Riemannian in general, including Riemannian. Thus, if $\varepsilon=1$, the signature of $g$ is equal to $2 p$, where $0 \leqslant p \leqslant n$; and if $\varepsilon=-1$, the signature of $g$ is equal to $2 p+1$, where $0 \leqslant p \leqslant n$.

When $\mu=1$, then the manifold $M$ is an almost paracontact metric manifold. In this case, the metric $g$ is pseudo-Riemannian, and its signature is equal to $n$ when $\varepsilon=1$, or $n+1$ when $\varepsilon=-1$. One notes that in this case, the eigenspaces of the linear operator $\varphi$ corresponding to the eigenvalues 1 and -1 are both $n$-dimensional at every point of the manifold.

\section{The Schouten-van Kampen connection adapted to an almost (para) contact metric structure}

5.1. Certain general conclusions. Let $M$ an almost (para) contact metric manifold, and consider the following pair of complementary and orthogonal distributions

$$
\mathcal{H}=\operatorname{ker} \eta, \quad \mathcal{V}=\operatorname{span}\{\xi\} .
$$

We have $\operatorname{dim} \mathcal{H}=2 n$ and $\operatorname{dim} \mathcal{V}=1$. $\mathcal{H}$ is usually called the contact or paracontact or canonical distributtion. We will call it a (para) contact distribution. 
The Schouten-van Kampen connection adapted to the pair (5.1) and arising from the Levi-Civita connection $\nabla$ will be called the Schouten-van Kampen connection adapted to the almost (para) contact metric structure, and will be denoted by $\widetilde{\nabla}$. As we have seen in Section 1 , the connection $\widetilde{\nabla}$ is given by $(2.4)$.

Now, by a direct calculation in which (2.4) should be used, we obtain the main formula for $\widetilde{\nabla} \varphi$.

Proposition 5.1. For an almost (para) contact metric manifold, we have

$$
\left(\widetilde{\nabla}_{X} \varphi\right) Y=\left(\nabla_{X} \varphi\right) Y+\eta(Y) \varphi \nabla_{X} \xi-\varepsilon g\left(\varphi \nabla_{X} \xi, Y\right) \xi
$$

As we can see from the above proposition, the condition

$$
\left(\nabla_{X} \varphi\right) Y=\varepsilon g\left(\varphi \nabla_{X} \xi, Y\right) \xi-\eta(Y) \varphi \nabla_{X} \xi
$$

is very important since it just means that $\widetilde{\nabla} \varphi=0$. The condition (5.3) will be used many times in the rest of the paper. The case $\operatorname{dim} M=3$ is the first situation where it occurs.

Proposition 5.2. For a 3-dimensional almost (para) contact metric manifold, the condition (5.3) is satisfied. Consequently, for a such a manifold, we have $\widetilde{\nabla} \varphi=0$.

Proof. The idea of the proof of the first assertion is precisely the same as that of [18, Proposition 1] and [31, Proposition 2.2]. Thus, we omit it. The second assertion follows now (5.3) and Proposition 5.1.

5.2. (Para) $\alpha$-contact metric manifolds. An almost (para) contact metric manifold will be called (para) $\alpha$-contact if $d \eta=\alpha \Phi$ for a certain non-zero function $\alpha$; and (para) $K$ - $\alpha$-contact if it is (para) $\alpha$-contact and $\xi$ is additionally a Killing vector field; cf. [16]. In the case when $\alpha=1$, we have a (para) contact metric manifold and a (para) $K$-contact manifold, respectively.

Proposition 5.3. An almost (para) contact metric manifold is

(a) (para) $\alpha$-contact if and only if $L-\varepsilon \alpha \varphi$ is a symmetric linear operator;

(b) (para) $K$ - $\alpha$-contact if and only if $L=\varepsilon \alpha \varphi$, where in the both above cases, $\alpha$ is a certain non-zero function.

Proof. (a) Note that by (3.2), we have

$$
\begin{gathered}
g((L-\varepsilon \alpha \varphi) X, Y)-g((L-\varepsilon \alpha \varphi) Y, X) \\
=\varepsilon\left(2 \alpha \Phi(X, Y)-\left(\nabla_{X} \eta\right)(Y)+\left(\nabla_{Y} \eta\right)(X)\right) \\
=2 \varepsilon(\alpha \Phi(X, Y)-d \eta(X, Y)) .
\end{gathered}
$$

Thus, $d \eta=\alpha \Phi$ if and only if $L-\varepsilon \alpha \varphi$ is a symmetric linear operator.

(b) Note that $\xi$ is Killing if and only if $L$ is a skew-symmetric linear operator, or equivalently $L-\varepsilon \alpha \varphi$ is a skew-symmetric linear operator. This constatation together with (a) gives our assertion (b).

Many curvature properties of the Schouten-van Kampen connections on contact or $K$-contact manifolds $(\varepsilon=1, \mu=-1)$ with positive definite metric were achived in $[\mathbf{2 7}, \mathbf{2 8}]$. 
5.3. Normal almost (para) contact metric manifolds. An almost (para) contact metric manifold (structure) will be called normal $([\mathbf{6}, \mathbf{1 3}])$ if the almost (para) complex structure $J$ defined on $M \times \mathbb{R}$ by

$$
J\left(X, a \frac{\partial}{\partial t}\right)=\left(\varphi X+\mu a \xi, \eta(X) \frac{\partial}{\partial t}\right)
$$

is integrable, or equivalently

$$
[\varphi, \varphi](X, Y)-2 \mu d \eta(X, Y) \xi=0,
$$

$[\varphi, \varphi]$ being the Nijehuis torsion tensor of $\varphi$, defined by

$$
[\varphi, \varphi](X, Y)=[\varphi X, \varphi Y]-\varphi[X, \varphi Y]-\varphi[\varphi X, Y]+\varphi^{2}[X, Y] .
$$

Note that if $\mu=-1$, then $J$ is an almost complex structure, and if $\mu=1$, then $J$ is an almost paracomplex structure.

Proposition 5.4. An almost (para) contact metric manifold is normal if and only if the shape operator $L$ commutes with $\varphi$ and

$$
\left(\widetilde{\nabla}_{\varphi X} \varphi\right) \varphi Y+\mu\left(\widetilde{\nabla}_{X} \varphi\right) Y=0 .
$$

Proof. Recalling [30, Lemma, p. 171] and [31, Proposition 2.1], we claim that the normality condition of an almost (para) contact structure can be formulated with the help of $\nabla \varphi$ in the following way

$$
\left(\nabla_{\varphi X} \varphi\right) Y-\varphi\left(\nabla_{X} \varphi\right) Y-\varepsilon \mu g\left(\nabla_{X} \xi, Y\right) \xi=0,
$$

or equivalently

$$
\left(\nabla_{\varphi X} \varphi\right) \varphi Y+\mu\left(\nabla_{X} \varphi\right) Y+\mu \eta(Y) \varphi \nabla_{X} \xi=0 .
$$

Note also that the normality condtion always implies

$$
\nabla_{\varphi X} \xi=\varphi \nabla_{X} \xi
$$

In fact, (5.7) follows easily from (5.5) when we put there $Y=\xi$. By (2.7), the relation (5.7) is equivalent to the commutativity of $L$ and $\varphi$, that is $L \varphi=\varphi L$.

Before we finish the proof, using (5.2), we find the following general formula for an arbitrary almost (para) contact metric manifold,

$$
\begin{array}{r}
\left(\widetilde{\nabla}_{\varphi X} \varphi\right) \varphi Y+\mu\left(\widetilde{\nabla}_{X} \varphi\right) Y=\left(\nabla_{\varphi X} \varphi\right) \varphi Y+\mu\left(\nabla_{X} \varphi\right) Y \\
+\mu \eta(Y) \varphi \nabla_{X} \xi+\varepsilon \mu g\left(\nabla_{\varphi X} \xi-\varphi \nabla_{X} \xi, Y\right) \xi
\end{array}
$$

If our almost (para) contact metric structure is normal, then applying (5.6) and (5.7) into (5.8), we obtain (5.4). mality.

If (5.4) and (5.7) hold, then from (5.8) we deduce (5.6), which gives the nor-

Proposition 5.5. For a 3-dimensional almost (para) contact metric manifold, the following conditions are equivalent:
(a) the manifold is normal,
(b) the shape operator $L$ commutes with $\varphi$,
(c) the shape operator is given by

$$
L X=\varepsilon \alpha \varphi X-\beta(X-\eta(X) \xi),
$$


$\alpha$ and $\beta$ being certain functions on $M$.

Proof. As we already know (see Proposition 5.2), $\widetilde{\nabla} \varphi=0$ for a 3-dimensional almost (para) contact metric manifold. Therefore, the equivalence (a) $\Leftrightarrow$ (b) follows from Proposition 5.4. It is obvious that $(\mathrm{c}) \Rightarrow(\mathrm{b})$. Finally, the implication (b) $\Rightarrow$ (c) can be easily verified when we use an adapted $\varphi$-basis $\left(e_{1}, e_{2}=\varphi e_{1}, e_{3}=\xi\right)$.

There is an additional differential equation related to the functions $\alpha$ and $\beta$ for an arbitrary 3-dimensional normal almost (para) contact manifold. To get it, using (5.9), we obtain

$$
\nabla_{X} \xi=-\varepsilon \alpha \varphi X+\beta(X-\eta(X) \xi)
$$

Hence,

$$
\left(\nabla_{X} \eta\right)(Y)=-\alpha g(\varphi X, Y)+\beta(\varepsilon g(X, Y)-\eta(X) \eta(Y)) .
$$

Moreover, using (5.10), from (5.3), we find

$$
\left(\nabla_{X} \varphi\right) Y=-\mu \alpha(g(X, Y) \xi-\varepsilon \eta(Y) X)+\beta(\varepsilon g(\varphi X, Y) \xi-\eta(Y) \varphi X) .
$$

Using (5.11) and (5.12), for the exterior derivatives of $\eta$ and $\Phi$, we get $d \eta=\alpha \Phi$ and $d \Phi=2 \beta \eta \wedge \Phi$. Therefore, $0=d^{2} \eta=(d \alpha+2 \alpha \beta \eta) \wedge \Phi$. Since $\operatorname{dim} M=3$, from the last equality, the following interesting equation follows

$$
d \alpha(\xi)+2 \alpha \beta=0 .
$$

In the next sections, we will study curvature properties of some subclasses of the class of normal almost (para) contact metric manifolds.

5.4. (Para) $\alpha$-Sasakian manifolds. We extend the notion of $\alpha$-Sasakian manifolds (see e.g. $[\mathbf{1 2}, \mathbf{6}, \mathbf{1 6}]$ ), and call an almost (para) contact metric manifold to be (para) $\alpha$-Sasakian if it satisfies the condition

$$
\left(\nabla_{X} \varphi\right) Y=-\mu \alpha(g(X, Y) \xi-\varepsilon \eta(Y) X)
$$

$\alpha$ being a function. Similarly as for $\alpha$-Sasakian manifolds, it can be proved that an almost (para) contact metric manifold is (para) $\alpha$-Sasakian if and only if it is normal and (para) $\alpha$-contact. As a consequence of (5.13), one obtains also $d \Phi=0$. Therefore, $0=d^{2} \eta=d \alpha \wedge \Phi$. Consequently, in dimensions $2 n+1 \geqslant 5$, it must be that $d \alpha=0$, that is, $\alpha$ is constant.

Proposition 5.6. An almost (para) contact metric manifold is (para) $\alpha$ Sasakian if and only if it is (para) $K$ - $\alpha$-contact and $\widetilde{\nabla} \varphi=0$.

PROOF. It is a straighforward verification that the condtion (5.13) is fulfilled if and only if the condtions (5.3) and

$$
\nabla_{X} \xi=-\varepsilon \alpha \varphi X
$$

hold simultanously. By virtue of $(5.2)$, the condition (5.3) is equivalent to $\widetilde{\nabla} \varphi=$ 0 . And, the condition (5.14) means that the manifold is (para) $K$ - $\alpha$-contact; see Proposition 5.3. 
Using the formula (3.4), we describe the relations between the curvatures of the Levi-Civita and the Schouten-van Kampen connections for (para) $\alpha$-Sasakian manifolds in dimensions $2 n+1 \geqslant 5$. But at first, using $L Y=\varepsilon \alpha \varphi Y$ (which follows from (5.14)), and (2.7), (3.2), (5.13), we find

$$
\left(\nabla_{X} L\right) Y-\left(\nabla_{Y} L\right) X=\mu \alpha(\eta(Y) X-\eta(X) Y) .
$$

Having the above in mind, from (3.4), we obtain the following:

TheOrem 5.1. For an (para) $\alpha$-Sasakian manifold of dimension $2 n+1 \geqslant 5$, the Riemann curvatures $\widetilde{R}, R$, the Ricci curvatures $\widetilde{S}, S$, and the scalar curvatures $\widetilde{r}, r$ are related by the following formulas

$$
\begin{aligned}
\widetilde{R}(X, Y, Z, W)= & R(X, Y, Z, W)+\mu \alpha(g(X, W) \eta(Y)-g(Y, W) \eta(X)) \eta(Z) \\
& +\mu \alpha(g(Y, Z) \eta(X)-g(X, Z) \eta(Y)) \eta(W) \\
& +\varepsilon \alpha^{2}(g(X, \varphi W) g(Y, \varphi Z)-g(X, \varphi Z) g(Y, \varphi W)), \\
\widetilde{S}(Y, Z)= & S(Y, Z)+\varepsilon \mu \alpha(1-\alpha) g(Y, Z)+\mu \alpha(2 n-1+\alpha) \eta(Y) \eta(Z), \\
\widetilde{r}= & r+2 n \varepsilon \mu \alpha(2-\alpha) .
\end{aligned}
$$

Corollary 5.1. For a (para) $\alpha$-Sasakian manifold of dimension $2 n+1 \geqslant 5$, the sectional curvatures curvatures $\widetilde{K}, K$ of a nondegenerate section $\sigma$ are related by the formulas

$$
\begin{aligned}
& \widetilde{K}(\sigma)=K(\sigma)+\varepsilon \alpha^{2} \quad \text { when } \sigma \text { is a } \varphi \text {-section, } \\
& \widetilde{K}(\sigma)=K(\sigma)+\varepsilon \mu \alpha \quad \text { when } \quad \xi \in \sigma .
\end{aligned}
$$

5.5. (Para) $\beta$-Kemotsu manifolds. Extending the notion of $\beta$-Kenmotsu manifolds (cf. $[\mathbf{1 2}, \mathbf{1 9}, \mathbf{9}]$, etc.), we define an almost (para) contact metric manifold to be (para) $\beta$-Kenmotsu if

$$
\left(\nabla_{X} \varphi\right) Y=\beta(\varepsilon g(\varphi X, Y) \xi-\eta(Y) \varphi X),
$$

$\beta$ being a function on $M$. Similar as for $\beta$-Kenmotsu manifolds, it can be proved that an almost (para) contact metric manifold is (para) $\beta$-Kenmotsu if and only if it is normal and

$$
d \Phi=2 \beta \eta \wedge \Phi, \quad d \eta=0 .
$$

Note that (5.16) implies $0=d^{2} \Phi=2 d \beta \wedge \eta \wedge \Phi$. Hence, in dimensions $2 n+1 \geqslant 5$, we have $\beta \wedge \eta=0$, by pure algebraic reasons. Coensequently, $d \beta=d \beta(\xi) \eta$. Denoting $\beta^{\prime}=d \beta(\xi)=\xi(\beta)$, we will write $d \beta=\beta^{\prime} \eta$. This is a strong restriction for the function $\beta$ in those dimensions.

Proposition 5.7. An almost (para) contact metric manifold is (para) $\beta$-Kenmotsu if and only if $\widetilde{\nabla} \varphi=0$ and

$$
L=\beta(-I+\xi \otimes \eta) .
$$

Proof. It is a straighforward verification that the condtion (5.15) is fulfilled if and only if the two condtions (5.3) and

$$
\nabla_{X} \xi=\beta(X-\eta(X) \xi)
$$


hold simultanously. By (5.2), the condition (5.3) is equivalent to $\widetilde{\nabla} \varphi=0$. And, by (2.7), the condition (5.18) is equivalent to (5.17).

We describe the relations between the curvatures of the Levi-Civita and the Schouten-van Kampen connections for a (para) Kenmotsu manifold in dimensions $2 n+1 \geqslant 5$.

As previously, we use the general formula (3.4). But at first, using (5.17), (2.7) and (3.2), we find

$$
\left(\nabla_{X} L\right) Y-\left(\nabla_{Y} L\right) X=-\left(\beta^{\prime}+\beta^{2}\right)(\eta(X) Y-\eta(Y) X) .
$$

Having the above in mind, from (3.4), we obtain the following:

TheOrem 5.2. For a (para) $\beta$-Kenmotsu manifold of dimension $2 n+1 \geqslant 5$, the Riemann curvatures $\widetilde{R}, R$, the Ricci curvatures $\widetilde{S}, S$, and the scalar curvatures $\widetilde{r}, r$ are related by the formulas

$$
\begin{aligned}
\widetilde{R}(X, Y, Z, W)= & R(X, Y, Z, W)+\varepsilon \beta^{2}(g(X, W) g(Y, Z)-g(X, Z) g(Y, W)) \\
& +\beta^{\prime}(\eta(X) \eta(W) g(Y, Z)-\eta(X) \eta(Z) g(Y, W) \\
& -\eta(Y) \eta(W) g(X, Z)+\eta(Y) \eta(Z) g(X, W)), \\
\widetilde{S}(Y, Z)= & S(Y, Z)+\varepsilon\left(\beta^{\prime}+2 n \beta^{2}\right) g(Y, Z)+(2 n-1) \beta^{\prime} \eta(Y) \eta(Z), \\
\widetilde{r}= & r+2 n(2 n+1) \varepsilon \beta^{2}+4 n \varepsilon \beta^{\prime} .
\end{aligned}
$$

Corollary 5.2. For a (para) $\beta$-Kenmotsu manifold of dimension $2 n+1 \geqslant 5$, the sectional curvatures curvatures $\widetilde{K}, K$ of a nondegenerate section $\sigma$ are related by the formulas

$$
\begin{aligned}
& \widetilde{K}(\sigma)=K(\sigma)+\varepsilon \beta^{2} \quad \text { when } \sigma \perp \xi, \\
& \widetilde{K}(\sigma)=K(\sigma)+\varepsilon\left(\beta^{\prime}+\beta^{2}\right) \text { when } \xi \in \sigma .
\end{aligned}
$$

5.6. (Para) trans-Sasakian manifolds. Consider a special subclass of almost (para) contact metric manifolds. Namely, those which satisfy the condtion

$$
\left(\nabla_{X} \varphi\right) Y=-\mu \alpha(g(X, Y) \xi-\varepsilon \eta(Y) X)+\beta(\varepsilon g(\varphi X, Y) \xi-\eta(Y) \varphi X),
$$

where $\alpha$ and $\beta$ are certain functions on $M$. Let us call such manifolds to be (para) trans-Sasakian. Similarly as for trans-Sasakian manifolds, it can proved that an almost (para) contact metric manifold is (para) trans-Sasakian if and only if it is normal and

$$
d \Phi=2 \beta \eta \wedge \Phi, \quad d \eta=\alpha \Phi
$$

REMARK 5.1. The above class of manifolds seems to be a natural generalization of the class of trans-Sasakian manifolds defined in [20], and since then, studied in many papers. It is important that in dimensions $\geqslant 5$, the class of trans-Sasakian manifolds splits into two subclasses: $\alpha$-Sasakian manifolds and $\beta$-Kenmostu manifolds, and contrary to that, in dimension 3, we do not have such a splitting; see [17]. Moreover, it is worth to notice that from [18, Propositions 1 and 2] it can be easily deduced the following (one has only to change the role of the functions $\alpha$ 
and $\beta$ ): In dimension 3 , the class of trans-Sasakian manifolds is precisely the class of normal almost contact metric manifolds. This fact was also mentioned in [1].

The following proposition is a generalization of the facts known for transSasakian manifolds.

\section{Proposition 5.8.}

(a) In dimension 3, the class of (para) trans-Sasakian manifolds coincides with the class of normal almost (para) contact metric manifolds.

(b) In dimensions $\geqslant 5$, the class of (para) trans-Sasakian manifolds splits into two subclasses: (para) $\alpha$-Sasakian manifolds and (para) $\beta$-Kenmostu manifolds. The common part of these subclasses form the (para) cosymplectic manifolds ( that is, those for which $\nabla \varphi=0$ ).

Proof. (a) Let us assume that the dimension is equal to 3. As we already know, in this dimension, the relation (5.3) is fulfilled. Therefore, (5.19) holds if and only if the condition (5.10) is satisfied. This condition is the same as (5.9), which is equivalent to the normality of our structure.

(b) At first, the exterior differentiation of both of the relations (5.20) gives

$$
\begin{aligned}
& 0=d^{2} \Phi=2 d \beta \wedge \eta \wedge \Phi+2 \alpha \beta \Phi \wedge \Phi, \\
& 0=d^{2} \eta=(d \alpha+2 \alpha \beta \eta) \wedge \Phi .
\end{aligned}
$$

Let us assume that the dimension is $\geqslant 5$. From (5.21), it follows that $\alpha \beta=0$. Therefore, from (5.22), we obtain $d \alpha=0$, that is, $\alpha$ is constant. Thus, we have got the first assertion.

\section{References}

1. F. R. Al-Solamy, J.-S. Kim and M. M. Tripathi, On $\eta$-Einstein trans-Sasakian manifolds, An. Sţiinţ. Univ. Al. I. Cuza Iaşi, Ser. Nouă, Mat. 57 (2011), No. 2, 417-440.

2. K. Arslan, C. Murathan, K. Olszak and Z. Olszak, On almost Hermitian structures induced on leaves of the canonical foliation on certain almost contact metric manifolds, Proceedings of the XV International Workshop on Geometry and Physics, Puerto de la Cruz, Tenerife, Canary Islands, Spain, September 11-16, 2006 Publ. de la RSME, Vol. 11 (2007), 216-222.

3. A. Bajancu, Kähler contact distributions, J. Geom. Phys. 60 (2010), 1958-1967.

4. A. Bejancu and H. Faran, Foliations and geometric structures, Mathematics and Its Applications Vol. 580, Springer, Dordrecht, 2006.

5. A. Bejancu and H. Faran, Curvature of the contact distribution, Publ. Math. Debrecen 76 (2010), No. 1-2, 1-20.

6. D. E. Blair, Riemannian Geometry of Contact and Symplectic Manifolds, Progress in Mathematics Vol. 203, Birkhäuser, Boston, 2002.

7. A. Bonome, R. Castro, E. Garcìa-Rìo and L. Hervella, Curvature of indefinite almost contact manifolds, J. Geom. 58 (1997), 66-86.

8. C. P. Boyer and K. Galicki, Sasakian geometry, Oxford Mathematical Monographs; Oxford Science Publications. Oxford: Oxford University Press (2008).

9. C. Călin and M. Crasmareanu, From the Eisenhart problem to Ricci solitons in f-Kenmotsu manifolds, Bull. Malays. Math. Sci. Soc. (2) 33 (2010), No. 3, 361-368.

10. S. Erdem, On almost (para) contact (hyperbolic) metric manifolds and harmonicity of $\left(\varphi, \varphi^{\prime}\right)-$ holomorphic maps between them, Houston J. Math. 28 (2008), No. 1, 21-45.

11. S. Ianuş, Some almost product structures on manifolds with linear connection, Kōdai Math. Sem. Rep. 23 (1971), 305-310. 
12. D. Janssens and L. Vanhecke, Almost contact structures and curvature tensors, Kodai Math. J. 4 (1981), 1-27.

13. S. Kaneyuki and F. L. Willams, Almost paracontact and parahodge structures on manifolds, Nagoya Math. J. 99 (1985), 173-187.

14. T. W. Kim and H.K. Pak, Canonical foliations of certain classes of almost contact metric structures, Acta Math. Sinica, Engl. Ser., 21 (2005), No. 4, 841-846.

15. V.F. Kirichenko and I. P. Borisovskiř, Integral manifolds of contact distributions (Russian), Mat. Sb. 189 (1998), No. 12, 119-134; translated in Sb. Math. 189 (1998), No. 12, 1855-1870.

16. V.F. Kirichenko and E. V. Rodina, On the geometry of trans-Sasakian and almost transSasakian manifolds (Russian), Fundam. Prikl. Mat. 3 (1997), No. 3, 837-846.

17. J. C. Marrero, The local structure of trans-Sasakian manifolds, Ann. Mat. Pura Appl. (IV) 162 (1992), 77-86.

18. Z. Olszak, Normal almost contact metric manifolds of dimension three, Ann. Math. Pol. 47 (1986), 41-50

19. Z. Olszak and R. Roşca, Normal locally conformal almost cosymplectic manifolds, Publ. Math. Debrecen 39 (1991), No. 3-4, 315-323.

20. J. A. Oubiña, New classes of almost contact metric structures, Publ. Math. (Debrecen) 32 (1985), 187-193

21. K. Sakamoto and Y. Takemura, On almost contact structures belonging to a CR-structure, Kodai Math. J. 3 (1980), 144-161.

22. J. Schouten and E. van Kampen, Zur Einbettungs- und Krümmungstheorie nichtholonomer Gebilde, Math. Ann. 103 (1930), 752-783.

23. A. F. Solov'ev, On the curvature of the connection induced on a hyperdistribution in a Riemannian space (Russian), Geom. Sb. 19 (1978), 12-23.

24. A. F. Solov'ev, The bending of hyperdistributions (Russian), Geom. Sb. 20 (1979), 101-112.

25. A. F. Solov'ev, Second fundamental form of a distribution (Russian), Mat. Zametki 31 (1982), 139-146; translated in Math. Notes 31 (1982), 71-75.

26. A. F. Solov'ev, Curvature of a distribution (Russian), Mat. Zametki 35 (1984), 111-124; translated in Math. Notes 35 (1984), 61-68.

27. A. F. Solov'ev, Curvature of a hyperdistribution and contact metric manifolds (Russian), Mat. Zametki 38 (1985), 450-462; translated in Math. Notes 38 (1985), 756-762.

28. A.F. Solov'ev, Hyperdistributions with parallel second fundamental form (Russian), Izv. Vyssh. Uchebn. Zaved., Mat. 289 (1986), No. 6, 21-29; translated in Sov. Math. 30 (1986), No. 6, 30-40.

29. T. Takahashi, Sasakian manifold with pseudo-Riemannian metric, Tôhoku Math. J. (2) 21 (1969), 644-653

30. S. Tanno, Almost complex structures in bundle spaces over almost contact manifolds, J. Math. Soc. Japan 17 (1965), 167-186

31. J. Wełyczko, On Legendre curves in 3-dimensional normal almost paracontact metric manifolds, Results Math. 54 (2009), 377-387.

32. S. Zamkovoy, Canonical connections on paracontact manifolds, Ann. Glob. Anal. Geom. 36 (2009), 37-60

Institute of Mathematics and Computer Science, WrocŁaw University of Technology, Wybrzeże Wyspiańskiego 27, 50-370 Wroceaw, Poland

E-mail address: zbigniew.olszak@pwr.wroc.pl 